\title{
A modality agnostic model for spatial resolution in mass spectrometry imaging: application to MALDI MSI data
}

Authors: Martin D. Metodiev ${ }^{\mathrm{a}, \mathrm{b}}$, Rory T. Steven ${ }^{\mathrm{a}}$, Xavier Loizeau ${ }^{\mathrm{a}}$, Zoltan Takats ${ }^{\mathrm{a}, \mathrm{c}}$, Josephine Bunch ${ }^{\mathrm{a}, \mathrm{b}, \mathrm{c}}$

a. National Centre of Excellence in Mass Spectrometry Imaging (NiCE-MSI), National Physical Laboratory (NPL), Teddington, UK, TW11 0LW

b. Imperial College London, Faculty of Medicine, Department of Metabolism, Digestion and Reproduction, London, UK, SW7 2AZ

c. Biological Mass Spectrometry, The Rosalind Franklin Institute, Harwell Campus, Didcot, UK, OX11 OFA

\section{Supporting Information Contents:}

- Section S1, page 2: provides information on deriving PSF/MTF/ESF equation; it shows results for MTF of an image with a low desorption rate.

- Section S2, page 5: provides information on how to calculate the resolution frequency and resolution number from data; the NPS and spectral cut-off are defined. Error analysis is discussed.

- Section S3, page 11: provides evidence of how resolution measurements vary with decreasing pixel size. The two main parameters affecting the resolution frequency are the MTF cut off and the blurring parameter.

- Section S4, page 12: encapsulates the resolution change across intensities. It contains graphs showing how the $86-14 \%$ criterion performs across intensities and a plot of SNR versus measured resolution.

- Section S5, page 13: contains further information on the analysis of resolution for tissue imaging 


\section{Section S1: Derivation of image formation from first principles}

A standard approach to describe image formation in MSI is a convolution based model $^{1} 2^{2} 3$ :

$$
\operatorname{image}(x, y)=\operatorname{PSF}(x, y) * \rho(x, y)
$$

Where $\operatorname{PSF}(x, y)$ is the point spread function - it is the image of a perfect point source and $\rho(x, y)$ is the molecular distribution over space- density; it is the object of the image. This model has long been used in SIMS imaging as a basis for a resolution measurement. The Fourier transform of equation $\mathrm{S} 1$ is no longer a convolution but a product:

$$
\mathcal{F}(\operatorname{image}(x, y))=\operatorname{MTF}(x, y) \mathcal{F}(\operatorname{object}(x, y))
$$

Where the MTF is the modulus of the Fourier Transform of the PSF. In one dimension it can be shown that a PSF is equivalent to a $\mathrm{LSF}^{2}$. Thus, the MTF can be obtained by Fourier transforming an LSF as well as a PSF in 1D. However, manufacturing a chemical point/line source is rather difficult an edge source is usually employed. Once a step edge is imaged, the LSF can be calculated as follows:

$$
\begin{aligned}
& \operatorname{LSF}(x)=\frac{\partial}{\partial x} \operatorname{ESF}(x) \\
& \operatorname{MTF}(x)=\mathcal{F}(\operatorname{LSF}(x))
\end{aligned}
$$

Where equations (S3) and (S4) show that an MTF can be computed from the image of an edge instead of using a grating-like device. The PSF (and hence the MTF) has so far been approximated by the shape of the analytical beam used to probe over the surface. In all reports thus far a stationary beam $I(x)$ is used to describe image formation. This implies that the analytical beam never moves and just dwells over the same spot. With this in mind, we now set up an imaging model which takes into account material consumption and discretization effects.

Consider the flux of ions $\phi_{i}$ of the $\mathrm{i}^{\text {th }}$ molecular density emanating from the sample surface. The total amount of ions that a mass spectrometer would collect in a pixel between the $n^{\text {th }}$ and the $(n+1)^{t h}$ scan will be:

$$
M_{i, n}=\int_{n \Delta t_{s c a n}}^{(n+1) \Delta t_{s c a n}} \phi_{i}(x, t) d t
$$

Where $\Delta t_{\text {scan }}$ is the scan time used to create the spectrum in each pixel. The flux of particles from the surface is linked to the molecular density by the Law of Conservation of mass:

$$
\phi_{i}=\frac{\partial}{\partial t} \gamma_{i} \int_{-\infty}^{\infty} \rho(x, t) d x=\int_{-\infty}^{\infty} \gamma_{i} \frac{\partial}{\partial t} \rho_{i}(x, t)+\rho_{i}(x, y) \frac{\partial}{\partial t} \gamma_{i} d x
$$

Where $\gamma_{i}$ is the ionisation probability of the $\mathrm{i}^{\text {th }}$ molecule. The term $\rho_{i}(x, y) \frac{\partial}{\partial t} \gamma_{i}$ corresponds to changes in the ionisation probability with respect to time. Such changes may be induced in a post-ionisation scheme. Another source of change for ionisation probability is moving to a different spatial location where the local chemical concertation of different molecules changes. Because molecules compete for charge in a set of chemical reactions, changing the analyte densities at a different spatial location will induce a change in the ionisation 
probability. This is referred to as the matrix effect. To simplify the problem at hand we neglect post ionisation and matrix effects by setting the change of the ionisation probability over time to be zero $\frac{\partial}{\partial t} \gamma_{i}=0$. Thus, we assume that a given molecule will have the same ionisation probability at all points of the sample of interest. It will always also be constant during which the image is recorded. This assumption should hold true for a homogeneous step edge, however that may not be the case in tissue imaging. Since $\gamma_{i}$ is now a constant, equation (S. 6) becomes:

$$
\phi_{i}=\gamma_{i} \int_{-\infty}^{\infty} \frac{\partial}{\partial t} \rho_{i}(x, t) d x
$$

Now, an expression for the evolution of the molecular density of the $i^{\text {th }}$ molecule is needed. An assumption is now made - all the desorbed particles leave the surface due to the presence of the analytical probe $I(x-v t)^{4}$ and the probe moves over the surface with a given uniform velocity $v_{\text {stage }}$; acceleration effects are neglected.

$$
\frac{\partial}{\partial t} \rho_{i}(x, t)=-k_{i} I(x-v t) \rho(x, t)
$$

Where $k_{i}$ is a desorption coefficient; it describes the probability of the $\mathrm{i}^{\text {th }}$ molecule to be desorbed under the influence of the moving analytical beam $I(x-v t)$. Such a desorption coefficient appears in the thermodynamic model of MALDI ion formation where the signal intensity is assumed to be proportional to a desorption coefficient, albeit $k_{i}$ is different here ${ }^{5}$. Yet a different desorption coefficient appears in the thermal proton transfer model of MALDI ${ }^{6}$. Of course, the approach taken here is qualitative whereas ${ }^{5}$ and ${ }^{6}$ are quantitative models of ion formation in MALDI.

Combining equations S7 and S8 and calculating the signal obtained in a pixel from equation S5 it can be seen that:

$$
M_{i, n}=\gamma_{i} k_{i} I *_{x} \rho_{i}(v t, t) *_{t} \operatorname{rect}\left(\frac{t}{\Delta t}-\frac{2 n+1}{2}\right)
$$

Note that the convolution over space assumes that $I(x-v t)=I(v t-x)$. In other words, the beam is symmetric. In this study we have picked a Gaussian beam profile which obeys that assumption. We can obtain a spot mode version of equation (S9). This is done by setting the stage velocity to approach zero over the scan time of a pixel. Substituting a point-mass density described by a Dirac delta function $\rho_{i}=\delta_{i}$ allows us to obtain a PSF. The results from a high desorption rate can be seen in the main article. The results from a low desorption rate are presented in Figure S1. These two scenarios can correspond to MALDI, where the matrix material is fully consumed and DESI or SIMS where the analyte distribution is not fully consumed as the analytical beam swipes over the tissue surface. 
(a)

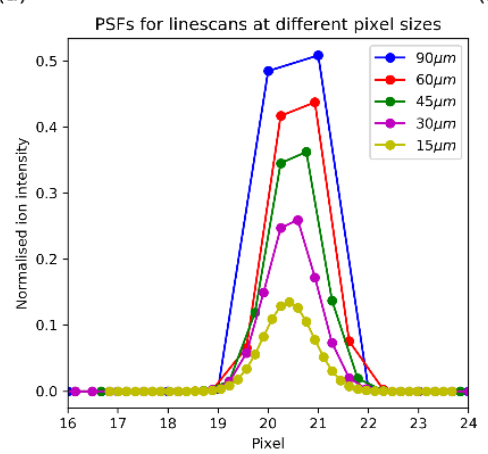

(b)

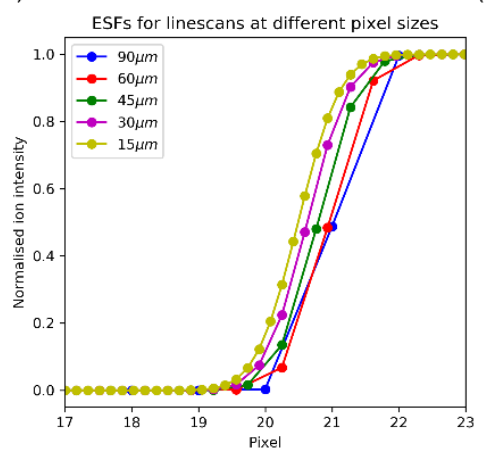

(c)

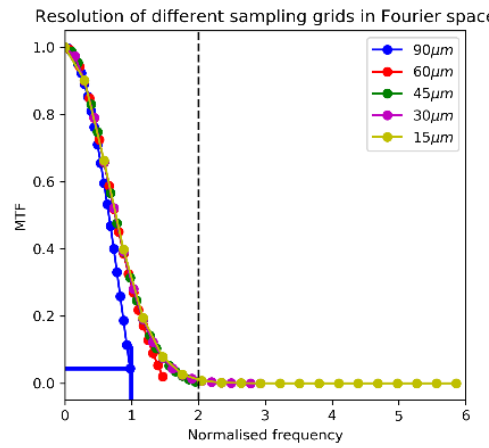

Figure S1. (a) and (b) show the PSF and ESF estimate for a point / edge source that is nonvanishing - material is not fully consumed as the beam sweeps over to new pixels and thus signal from previous pixels is detected. (c) shows the MTF calculation for a non-decaying point source - it vanishes at a normalised frequency twice the Nyquist frequency of $90 \mu \mathrm{m}$ grid, which corresponds to $90 \mu \mathrm{m}$. Thus, resolution is lost at the beam width due to a blur.

In Figure S1 it is demonstrated that when oversampling occurs in the case of weak sample consumption rates (SIMS/DESI), resolution is saturated at the beam width.

(a)

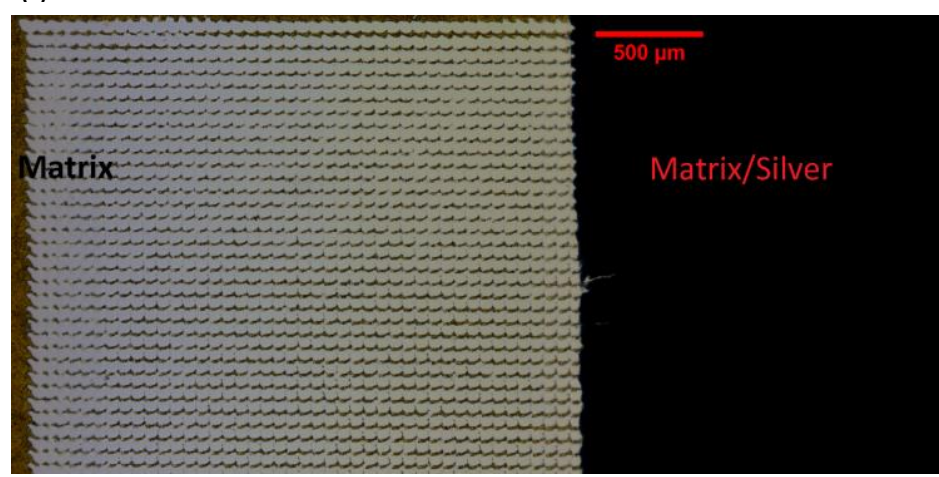

(b) 2d ESF at $75 \mu \mathrm{m}$

$[M+A g]^{+}$

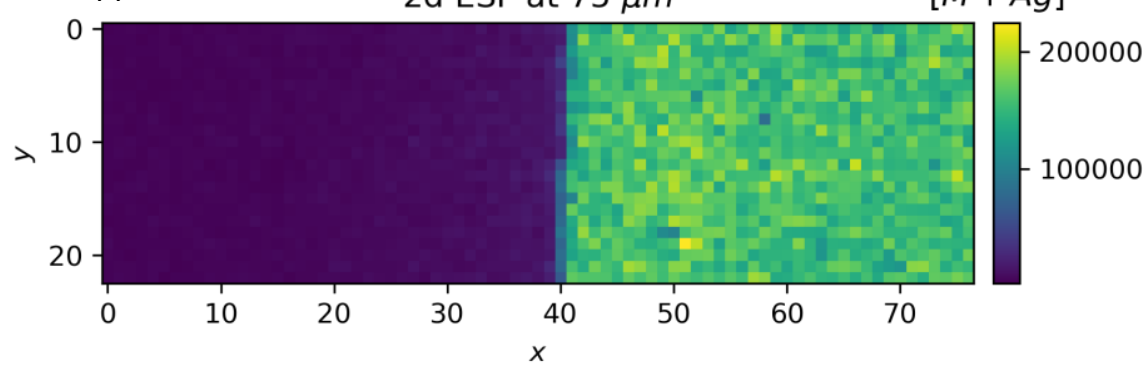

Figure S2. (a) a microscopy image of a silver step edge coated with matrix sampled at 75micron pixel size. (b) an MSI single ion image representing the matrix and silver ion adduct at 75 microns. 
Figure $\mathrm{S} 2$ shows a brightfield transmission microscopy image of the silver and matrix system. The silver and matrix region (right hand side) is not visible as light is blocked by the silver layer. The matrix region (left hand side) is covered with laser ablation craters. The laser spot size was measured to be around 85 microns. The MSI image on the bottom represents the most intense peak in a 75 microns data set. There is a slight amount of oversampling (crater overlapping) in the $\mathrm{x}$ direction. The two-dimensional edge image is then averaged over in the $\mathrm{y}$ direction to produce an average ESF.

\section{Section S2: Noise modelling and error analysis}

This section is dedicated to establishing an appropriate noise model for MSI and also providing an uncertainty band for the resolution point.

\section{Section 2.1: Calculating the MTF and the SNR}

The MTF is straightforward to calculate. This can be done from the signal using:

$$
M T F=|\mathcal{F}(L S F)|=\left|\mathcal{F}\left(\frac{\partial}{\partial x}<E S F>\right)\right|
$$

Therefore, the resolution point $f_{c}$ is defined as the point of intersection between MTF and NPS. The resolution distance $R$ can be calculated using:

$$
d=p \frac{2}{f_{c}}
$$

Where $p$ is the pixel size. The MTF cut-off is defined as:

$$
\operatorname{MTF} \text { cut off }(\%)=\frac{<N P S>}{M T F(0)} 100
$$

Where $\langle N P S>$ is the average of the noise power spectrum and $M T F(0)$ is the value of the MTF at the zeroth frequency. $M T F(0)$ should correspond to the average intensity of the edge surface.

We can compare NPS against an SNR metric.

$$
S N R=\frac{<\text { signal }>}{\frac{1}{\sqrt{n}} \sum_{i} \sqrt{\left(\text { signal }_{i}-<\text { signal }>\right)^{2}}}=\frac{<\text { signal }>}{\sigma(\text { noise })}
$$

Where $<$ signal $>$ is the signal average, $n$ is the number of linescans used to average over the signal, signal $_{i}$ is the signal of the $i^{\text {th }}$ linescan and $\sigma$ (noise) is the standard deviation of the estimated noise.

\section{Section S2.2: Noise modelling and scaling - potential issues noted}

When the noise function is computed empirically, the estimate will depend on the number of rows and columns in the image. We are interested in normalising the NPS in such a way that the final output represents the noise levels of a single linescan and not the average over all linescans, which would be a lower value. Thus, this is a scaling problem in the y direction. 
Furthermore, when an edge is used to obtain an LSF, the observed noise levels will increase. This is because the derivative of the signal is used to compute the LSF and the original noise in edge surface is carried over to the LSF. This is a scaling problem in the $\mathrm{x}$ direction.

In order to define resolution as a function of noise we need to quantify the amount of noise present in the signal. Previously, we had:

$$
M_{i, n}=\gamma_{i} k_{i} I *_{x} \rho_{i}(v t, t) *_{t} \operatorname{rect}\left(\frac{t}{\Delta t}-\frac{2 n+1}{2}\right)+\eta_{i}
$$

However, now we have introduced an additive noise parameter $\eta$. In the one-dimensional case of an image of a step edge we replace the left-hand side term of equation (S14) with a simple Gaussian CDF model, which we refer to as an edge spread function (ESF). Once we average over linescans $M_{n}$ the average signal becomes:

$$
<\text { signal }>=<E S F+\frac{1}{n} \sum_{i}^{n} \eta_{i}
$$

The noise level $\eta_{i}$ for an individual linescan $i$ may be computed as:

$$
\eta_{i}=<\text { signal }>- \text { signal }_{i}
$$

We assume that the expectation value of the noise is centred at zero. Thus, all negative and positive noise contributions will tend to cancel each other out. Therefore, the expected value of the noise is:

$$
\mathbb{E}\left[\eta_{i}\right]=0
$$

And the variance can be expressed as:

$$
\mathbb{V}\left[\eta_{i}\right]=\mathbb{E}\left[\eta_{i}^{2}\right]=\frac{n-1}{n} \sigma^{2}
$$

The expected value of the Fourier Transform of the average of the noise will also be zero.

$$
\mathbb{E}\left[\mathcal{F}\left(\eta_{i}\right)\right]=0
$$

However, the expected value of the modulus of the Fourier Transform of the noise average will be:

$$
\mathbb{E}\left[\left|\mathcal{F}\left(\eta_{i}\right)\right|^{2}\right]=\mathbb{V}\left[\mathcal{F}\left(\eta_{i}\right)\right]+\mathbb{E}\left[\mathcal{F}\left(\eta_{i}\right)\right]=\frac{n-1}{n} \sigma^{2}
$$

Thus, we can define the NPS as:

$$
N P S=\frac{1}{\sqrt{n_{x}}\left(n_{y}-1\right)} \sum_{i} \sqrt{\left|\mathcal{F}\left(\eta_{i}\right)\right|^{2}}
$$

Thus, we can evaluate the Fourier Transform of the average amount of noise in a single linescan. The $\sqrt{n_{x}}$ is included in order to tackle the x scaling issue (see Figure S3) and will be discussed shortly. In order to show the scaling effect on the NPS estimate with number of linescans $\left(n_{y}\right)$ we plot the MTF cut-off (which is the ratio of the average of the NPS and the MTF at the zeroth frequency) against an increasing number of linescans in Figure S3: 


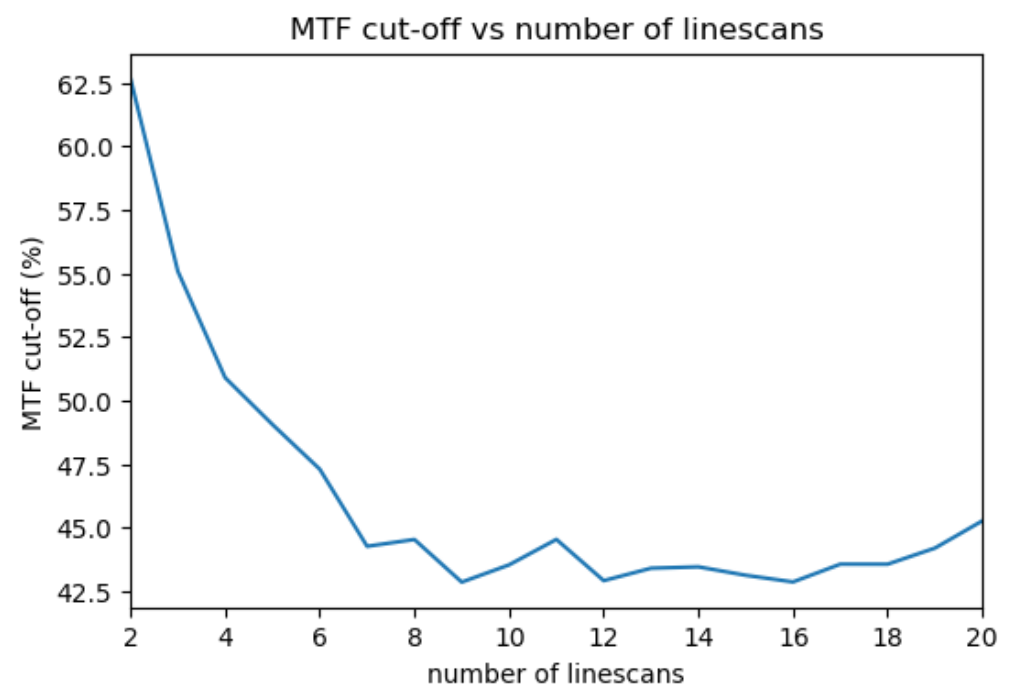

Figure S3 The number of linescans it takes to obtain a good average estimate of the NPS. Above 7-8 linescans the average begins to converge to a constant value. Thus, for this method to work an image with at least 5 linescans should be used.

Another approach to solving this problem is to assume that there is no correlation between the noise and the intensity. Then the NPS can be obtained simply from:

$$
N P S=\frac{\sqrt{n_{y}}}{\sqrt{n_{x}}} \mathcal{F}\left(<E S F>-\eta^{\prime}\right)
$$

Where $\eta^{\prime}$ is the noise of the average signal and $n_{y}$ is the number of linescans in the image and the $\sqrt{n_{x}}$ is related to $\mathrm{x}$ scaling. It should be noted that this method assumes that there is no correlation between intensity and noise and MSI data certainly has a noise-intensity correlation. In regions of the image where there is no signal the noise levels are quite small. Thus, if we select a part of an edge image which contains a lot more pixels without a signal equation S18 will tend to overestimate the average value of the NPS. On the other hand, if we select fewer empty pixels and more pixels containing a signal equation S22 acts like a good approximation. This problem is depicted in Figure S4. 

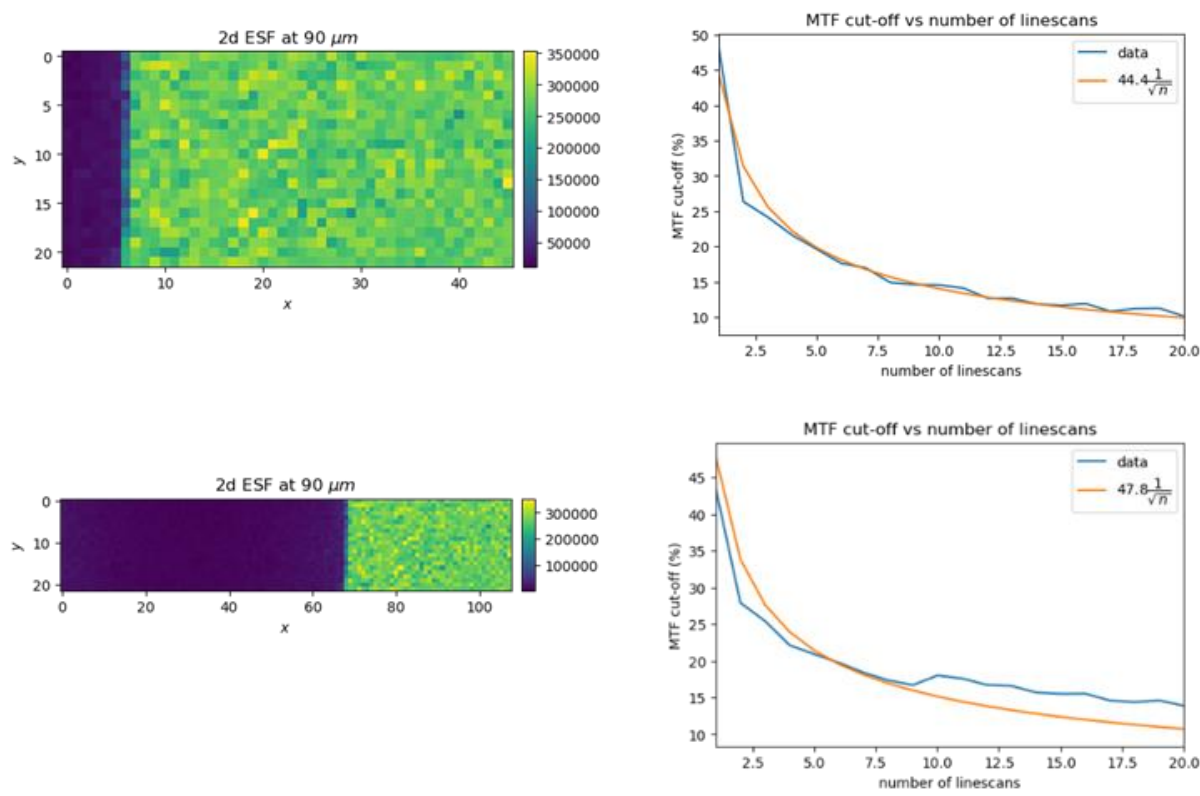

Figure S4. Top row - square root scaling when less pixels exhibit an intensity-noise correlation. Bottom row - square root scaling when more pixels exhibit an intensity-noise correlation.

Figure S4 depicts the same image when a different number of pixels is added. The graphs on the right-hand side show the scaling properties as the number of linescans are increased. In this case the number of linescans is increased from 1 to 23 . Because averaging over more linescans reduces the noise observed in the average of the signal the NPS value should be scaled to that of a single linescan. It can be argued that for a noise distribution which is independent of the intensity this scaling is proportional to the square root of the number of linescans. The graph on the top right-hand side shows that this holds well when less lower intensity pixels are included. However, the graph on the bottom right-hand side depicts what happens when more pixels that do not contain a signal from the edge are included. Using a square root as a scaling factor does not always work. This is because the assumption that the noise and the signal are not correlated is not true.

With all of this in mind we have chosen equation S21 as the definition of the NPS as it provides a more accurate estimate of the noise behaviour in a single line scan and it doesn't suffer from the intensity-noise independence assumption. The stability of this can be seen in Figure S3.

The issue of NPS scaling in the X-direction is now discussed. Figure S3 showed that adding more pixels in the $\mathrm{x}$ direction that do not contain a signal from the edge made the square root approximation when trying to scale in the y direction less accurate. This did not increase the noise level but instead the noise level deviated from the square root dependence. On the other hand, adding more pixels that contain a signal from the edge will increase the estimated noise level. This is because the approach described here uses an edge instead of a line source. The right-hand side of the LSF will contain more noise compared to the left-hand side (see Figures 2 (a) and (c) in the main article). One obvious way of avoiding it is to image a sharp 
line instead of an edge. Another way is to consider the relationship between number of pixels in the $\mathrm{x}$ direction and the MTF cut-off as depicted in Figure S5
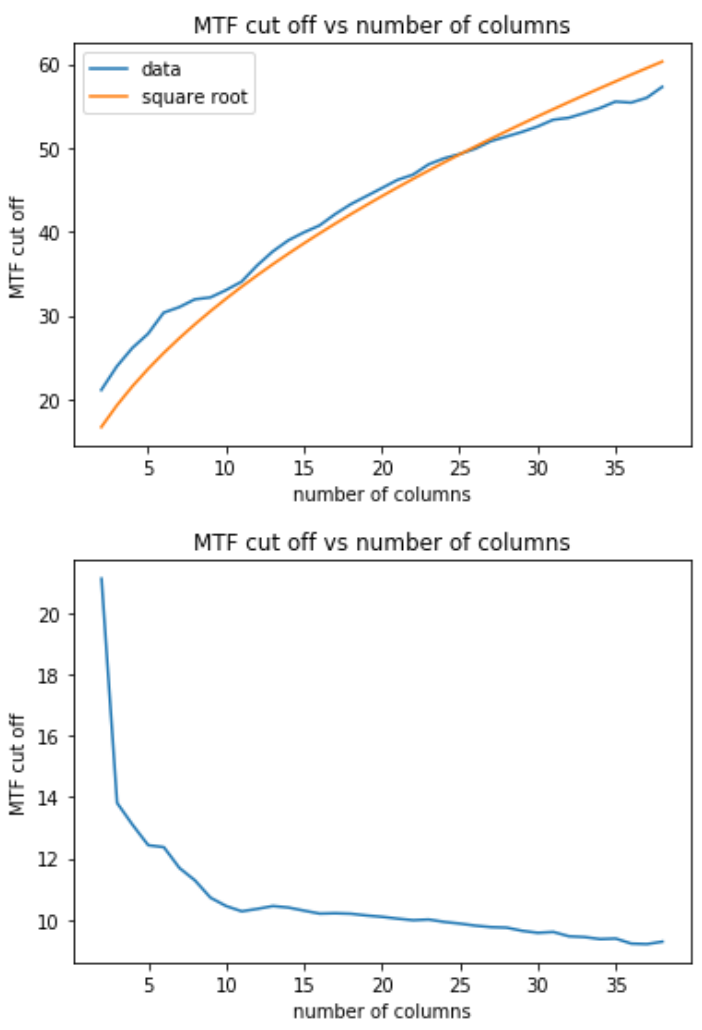

Figure S5. Top - noise increase with adding more columns (pixels in the x direction). Bottom - Noise normalised by $\sqrt{n_{x}}$

Here we have shown the increase of the estimated noise function from two pixels containing a signal from the edge to 38 pixels. The increase is approximately proportional to the square $\mathrm{f}$ $n_{x}$ - the number of pixels in the $\mathrm{x}$ direction that contain a signal from the edge surface. The square root term here is only an approximation, hence we can see that adding to many pixels may lead to underestimating the noise levels and therefore calculating a higher resolution number. The stability seen here is not as good as the one in Figure S3. The actual noise distribution function needs to be estimated in order to find an appropriate normalization factor. However, this is a topic for future work.

\section{Section S2.3: Error analysis}

The MTF is modelled with a Gaussian function centred at zero:

$$
M T F=A \exp \left(-\frac{f^{2}}{2 \sigma^{2}}\right)
$$


The parameters $A$ and $\sigma$ can be estimated using bootstrapping. Let $\delta A$ and $\delta \sigma$ represent the uncertainties derived from the bootstrap fit. We are interested in finding the point of intersection between the MTF and the average value of the NPS. Let the average value of the NPS be denoted by $k$ and let the standard error of $k$ be $\delta k$.

$$
\delta k=\frac{\sigma(k)}{\sqrt{n}}
$$

$\sigma(k)$ is the standard deviation of of $k$ and $n$ is the number of linescans.

We are interested in computing the error in the cut-off frequency $f_{c}$, which is the intersection between Equation $\mathrm{S} 23$ and $k$.

$$
\operatorname{Aexp}\left(-\frac{f^{2}}{2 \sigma^{2}}\right)=k
$$

Equation S25 is solved by:

$$
f_{c}= \pm \sqrt{2 \sigma^{2} \ln \left(\frac{A}{k}\right)}
$$

Where the negative solution can be neglected as it is non-physical. The uncertainty in $f_{c}, \delta f_{c}$ is given by:

$$
\delta f_{c}=\sqrt{\left(\frac{\partial f_{c}}{\partial \sigma}\right)^{2} \delta \sigma^{2}+\left(\frac{\delta f_{c}}{\delta A}\right)^{2} \delta A^{2}+\left(\frac{\partial f_{c}}{\partial k}\right)^{2} \delta k^{2}}
$$

After calculating the partial derivatives, we obtain:

$$
\delta f_{c}=\sqrt{\frac{\delta k^{2} \sigma^{2}}{2 k^{2} \ln \left(\frac{A}{k}\right)}+\frac{\delta A^{2} \sigma^{2}}{2 A^{2} \ln \left(\frac{A}{k}\right)}+2 \delta \sigma^{2} \ln \left(\frac{A}{k}\right)}
$$

Which is the error in the cut-off frequency. This can be propagated back to distance using:

$$
\delta d=d \sqrt{\left(\frac{\delta f_{c}}{f_{c}}\right)^{2}}
$$

\section{Section S3: Experimental results from different pixel sizes}


(a)

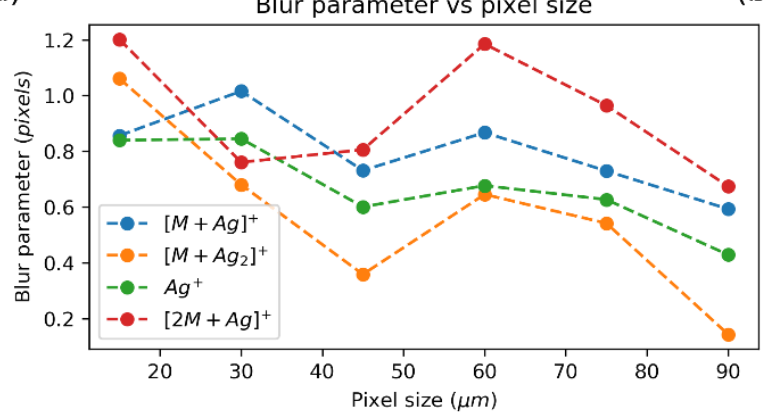

(b)

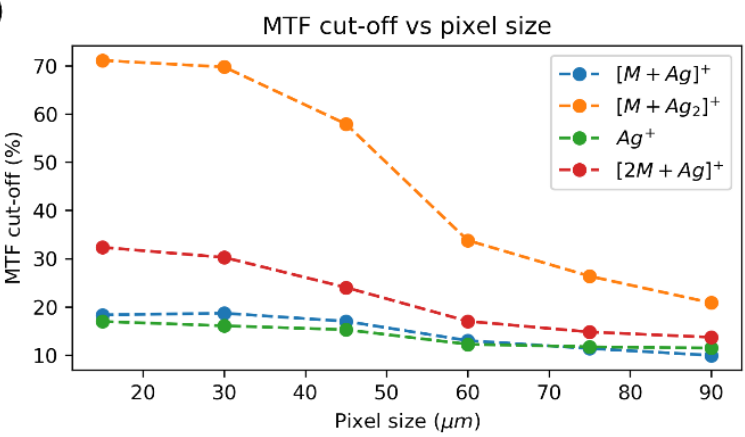

Figure S6. (a) blurring parameter estimate for the four different single ion images across different pixel sizes. From here a standard $86-14 \%$ is computed when multiplying blur values by a factor of 2 . On their own, most of these values violate the Sampling theorem if they were to be used as a resolution metric. (b) spectral cut-off values representing the percentage of total signal intensity at which NPS intersects MTF.

(a)

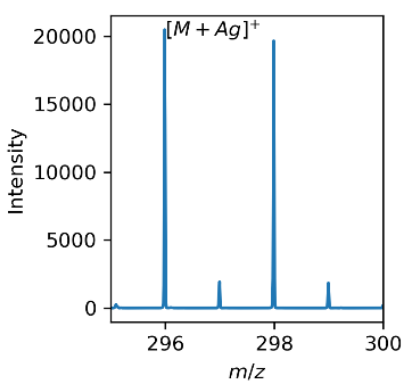

(e)

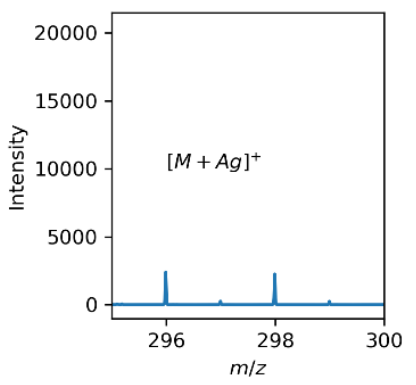

(b)

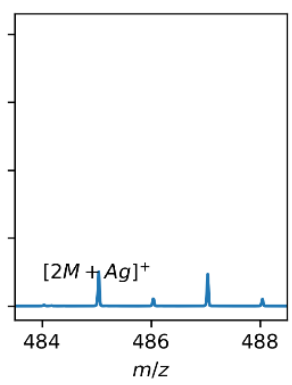

(f)

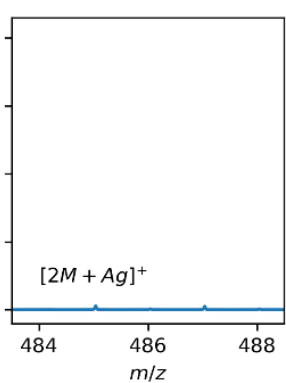

(c)

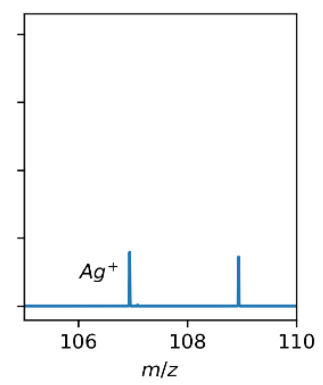

(g)

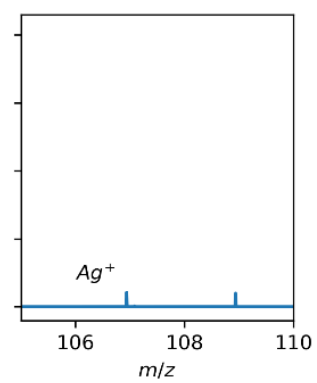

(d)

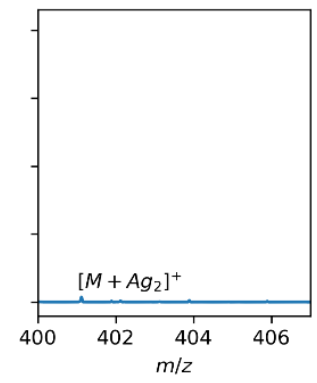

(h)

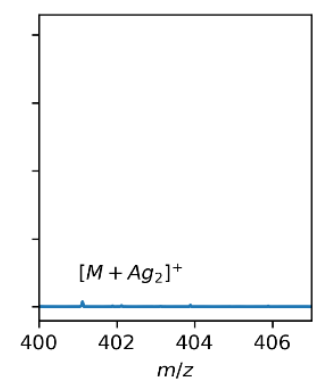

Figure S7. (a), (b), (c) and (d) The mean spectra of the $[M+A g]^{+}$and its isotopes, $[2 M+A g]^{+}$ion image and its isotopes, $\mathrm{Ag}^{+}$and its isotopes and the $\left[M+A g_{2}\right]^{+}$image at $90 \mu \mathrm{m}$. (e), (f), (g) and (h) The mean spectra of the same species at $15 \mu \mathrm{m}$

Signal intensity decreases with pixel size and noise becomes more significant. This behaviour can be seen across the columns of the figure. The resolution of lower intensity images at a given pixel size deteriorates with signal intensity. The above figure represents the spectra of the two end data points $(90 \mu \mathrm{m}$ and $15 \mu \mathrm{m})$ in Figure 3 (a).

\section{S4: Resolution change across intensity}


(a)

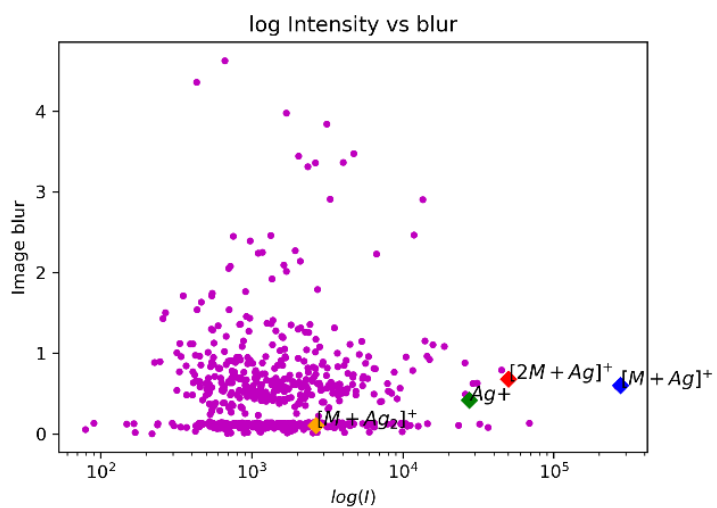

(b)

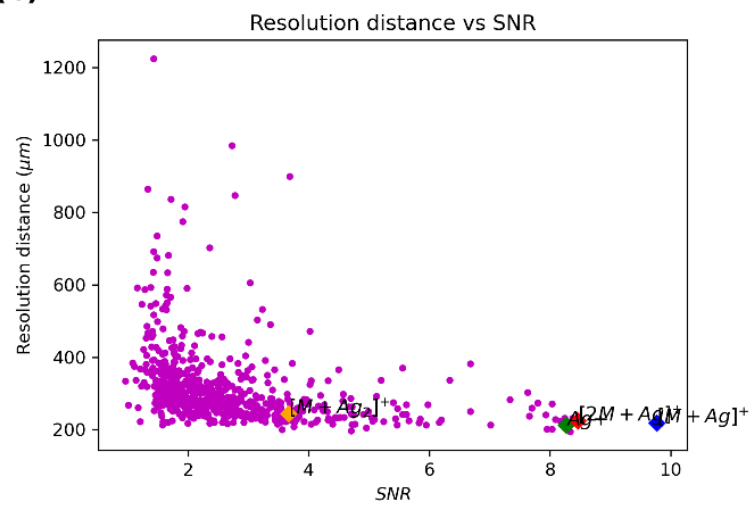

Figure S8. (a) The blurring parameter derived from the edge. (b) Resolution distance vs SNR

The values in Figure S8 (a) would yield the 86-14\% criterion if multiplied by two. Most of these values are below two pixels. It is interesting to point that there are two distinct clusters of image blur in the images.

Figure S8 (b) depicts the relationship between SNR and resolution distance. Images with lower SNR have a worse resolution whereas images with high SNR have a better resolution.

\section{S5: Resolution in tissue imaging}
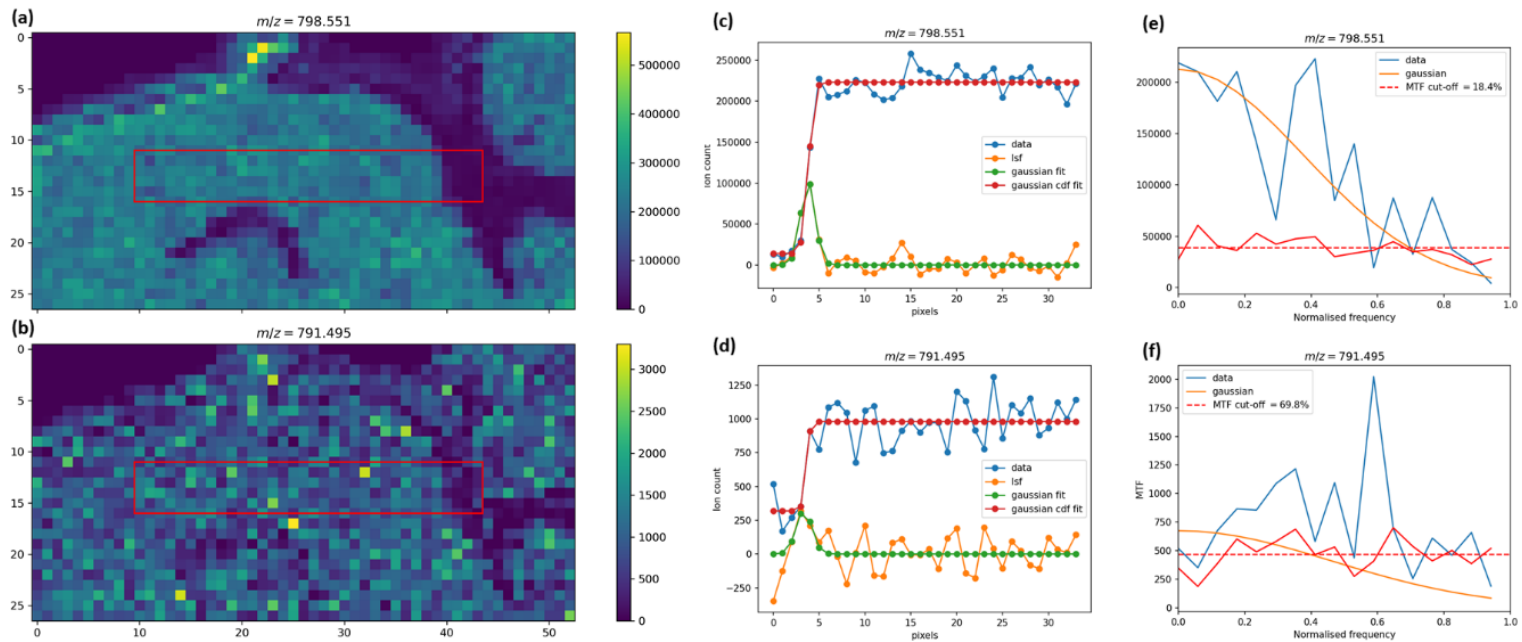

Figure S9. (a) and (b) - the region of interest used as a step edge for for the high intensity signal $m / z=798.551$ and the lower intensity signal $m / z=791.495$ is marked with a red box. The edge ROI consisted of 7 linescans each of which was 33 pixels in the $\mathrm{x}$ direction.

(c) and (d) - an ESF fit to the edge ROI of the high and low intensity ion images respectively.

(e) and (f) the MTF and NPS derived from the edge ROI of the high and low intensity ion images respectively. 
(a)

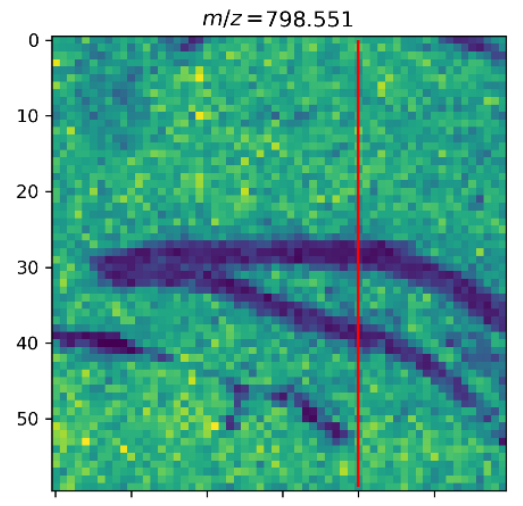

(b)

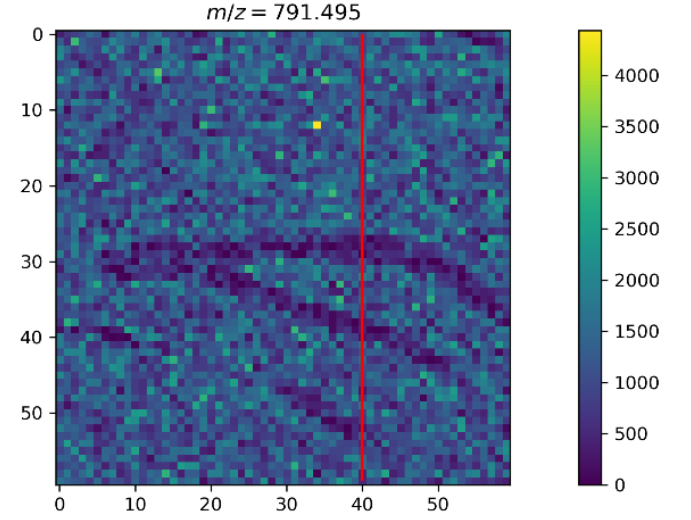

(c)

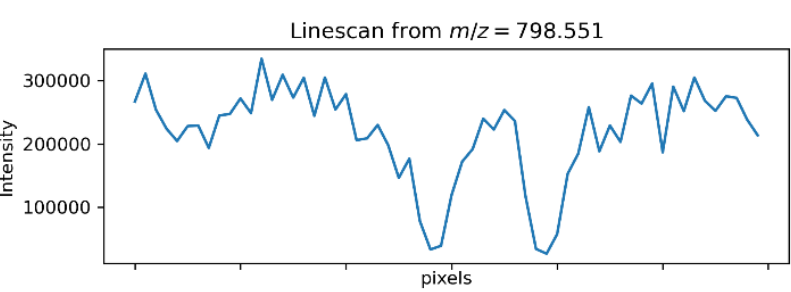

(d)

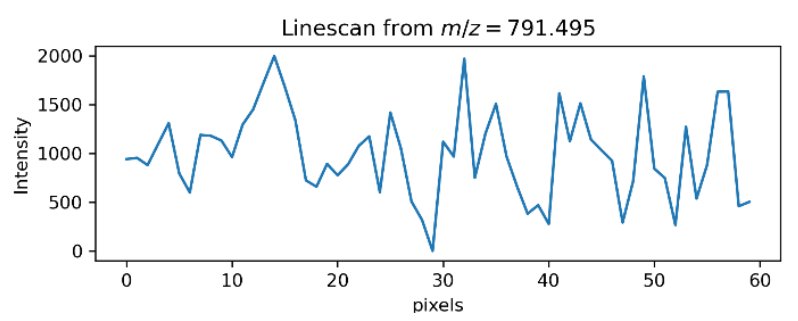

Figure S10. (a) and (b) A zoomed in version of an anatomical feature marked by an yellow box seen in Figure 5 of the main article for the high intensity signal $\mathrm{m} / \mathrm{z}=798.551$ and the lower intensity signal $\mathrm{m} / \mathrm{z}=791.495$. The mean intensity of the feature in both images is very close to the mean intensity of the edge ROIs in Figure S9, thus the resolution estimate is applicable. The continuous red line marks an example linescan. (c) and (d) An example linescan taken from an anatomical feature. The valleys in the high intensity image are resolved and the valleys in the lower intensity image can no longer be resolved.

\section{Bibliography}

1. Zubair F., Prentice B.M., Norris J.L., Laibinis P.E., Caprioli R. M. Standard Reticle Slide To Objectively Evaluate Spatial Resolution and Instrument Performance in Imaging Mass Spectrometry Anal. Chem., 2016, 88, 14, 7302-11.

2. Passarelli M.K., Wang J., Mohammadi A.S., Trouillon R., Gilmore I., Ewing A.G. Development of an organic lateral resolution test device for imaging mass spectrometry Anal. Chem., 2014, 86, 19, 9473-80.

3. Seah M.P. Resolution parameters for model functions used in surface analysis Surf. Interface Anal., 2002, 33, 12, 950-3

4. Jurchen J.C., Rubakhin S.S., Sweedler J.,V. J. MALDI-MS imaging of features smaller than the size of the laser beam Am. Soc. Mass. Spectrom., 2005, 16,10, 1654-9.

5. $\quad$ Lai Y.H., Wang C.C., Lin S.H., Lee Y.T., Wang Y.S. Solid-phase thermodynamic interpretation of ion desorption in matrix-assisted laser desorption/ionization J. Phys. Chem. B, 2010, 114, 43, 13847-52. 
6. Chu K.Y., Lee S., Tsai M.T., Lu I.C., Dyakov Y.A., Lai Y.H., Ni C.K. Thermal proton transfer reactions in ultraviolet matrix-assisted laser desorption/ionization J. Am. Soc. Mass. Spectrom., 2014, 25, 3, 10-8. 